\title{
The impact of different surface treatments on the shear bond strength of orthodontic metal brackets applied to different CAD/CAM composites
}

\author{
Roberto-Maia de Almeida ${ }^{1}$, Viviane Hass ${ }^{2}$, Debora-Yumi Sasaki ${ }^{3}$, Sandrine-Bittencourt Berger ${ }^{4}$, Thais-Maria \\ Fernandes ${ }^{4}$, Mateus-Rodrigues Tonetto ${ }^{5}$
}

\author{
${ }^{1}$ MSc Candidate, Postgraduate Program in Integrated Dental Science, School of Dentistry, University of Cuiaba-UNIC, Cuiaba, \\ MT, Brazil \\ ${ }^{2}$ Postdoctoral Fellow, University of Missouri-Kansas City, School of Dentistry, Kansas City, MO, USA \\ ${ }^{3}$ Undergraduate Student, School of Dentistry, University of North Parana - UNOPAR, Londrina, PR, Brazil \\ ${ }^{4}$ Associate Professor, Postgraduate Program in Dentistry, School of Dentistry, University of North Parana - UNOPAR, Londrina, \\ PR, Brazil \\ ${ }^{5}$ Associate Professor, Postgraduate Program in Integrated Dental Science, School of Dentistry, University of Cuiaba-UNIC, Cuia- \\ ba, MT, Brazil
}

Correspondence:

University of Missouri-Kansas City

School of Dentistry. 650 E. 25th St.

Kansas City, MO 64108, USA

viviklhass@hotmail.com

de Almeida RM, Hass V, Sasaki DY, Berger SB, Fernandes TM, Tonetto MR. The impact of different surface treatments on the shear bond strength of orthodontic metal brackets applied to different cad/cam composites. J Clin Exp Dent. 2021;13(6):e608-13.

\begin{tabular}{|l}
\hline Article Number: $58137 \quad$ http://www.medicinaoral.com/odo/indice.htm \\
(C) Medicina Oral S. L. C.I.F. B 96689336 - eISSN: 1989-5488 \\
eMail: jced@jced.es \\
Indexed in: \\
Pubmed \\
Pubmed Central $@$ (PMC) \\
Scopus \\
DOI@ System
\end{tabular}

\begin{abstract}
Background: To investigate the shear bond strength (SBS) of orthodontic metal brackets applied to different CAD/ CAM composites treated with different surface treatments.

Material and Methods: Specimens of two CAD/CAM composites were obtained of Lava Ultimate (LU; $n=60$ ) and Brilliant $C$ rios $(B C ; n=60)$ which were randomly separated into six subgroups $(n=10)$ according to the surface treatment: control (CTL); sandblasting (SB); sandblasting and silane (SBSL); hydrofluoric acid (HF); hydrofluoric acid and silane (HFSL); and Monobond Etch\&Prime (MEP). The mandibular central incisor metal brackets were bonded with a light-cure adhesive. The SBS data were analyzed using the two-way analysis of variance and Turkey's test, while the adhesive remnant index (ARI) by the Kruskal-Wallis, all the significance was set at 5\%.

Results: A higher SBS was found for BC in comparison with LU $(p<0.05)$. All the surface treatments increased the SBS in comparison with CTL $(p<0.0001)$. Treatment with HF, SBSL and HFSL $(p>0.05)$ showed a higher SBS, which was followed by MEP and SB $(p>0.05)$, all in comparison with CTL $(p<0.0001)$. For ARI, a significant effect was detected only for the surface treatment $(p<0.01)$, and not for CAD/CAM resin $(p>0.05)$. Significant differences were detected between CTL to HF, and HF to MEP, as well.

Conclusions: The SBS is highly affected by the surface treatment and also by the CAD/CAM composite. The surface treatment improves the SBS and should be encouraged when orthodontic brackets are bonded to CAD/CAM composites.
\end{abstract}

Key words: CAD/CAM composite resin, brackets, shear bond strength, surface treatment, bonding. 


\section{Introduction}

The CAD/CAM technology is widely growing in Dentistry because of their superior advantages compared to conventional procedures by the facilitated manufacturing process of indirect restorations: fast, convenient and minimizes the human factor (1). Likewise, novel CAD/ CAM composites have been introduced in the market with impressive evolution in terms of mechanical and aesthetic properties, which has encouraged a wide variety of clinical indications (2). Thus, a need for orthodontic treatment in patients with indirect restorations has also emerged and orthodontists frequently encounter the challenge of efficiently bonding orthodontic brackets to different CAD/CAM composites.

These materials consist of innovative microstructures that contain a polymeric matrix and dispersed fillers, which are associated with new polymerization modes. This implies in different characteristics and properties, and notably comparison with ceramic materials (2). Studies reported difficulties to bonding brackets on dental ceramics and pointed out the importance of additional surface treatments added on bonding procedures $(3,4)$. Etching with hydrofluoric acid or sandblasting, combined with silane application, are some of the most accepted surface treatments to bonding on dental ceramics $(2,5-8)$. To simplify this procedure, a novel ceramic pri- mer was developed, which combined the etching and primer in the same step (9) and showed promising results (5).

However, in contrast to dental ceramics, surface treatments and bonding protocols are still not well established for CAD/CAM composites $(2,6-8)$, probably because they are relatively novel materials with specific characteristics and significant variations among them. Especially when dealing with brackets bonding, only one study evaluated it on a CAD/CAM composite, and unfortunately a comparison among different CAD/CAM composites was not studied (10). Thus, there is crucial need to evaluate different CAD/CAM composites and different surface treatments. This in vitro study evaluated the effect of surface treatments on SBS of metal brackets applied to different CAD/CAM composites. The null hypotheses were that the SBS would not be affected by: 1) the different CAD/CAM composites and 2) surface treatments.

\section{Material and Methods}

-Preparation of the CAD/CAM blocks and surface treatment

Blocks of two CAD/CAM composites (Lava Ultimate-LU; and Brilliant Crios-BC; Table 1) were sectioned into rectangular specimens $(7 \times 7 \times 5 \mathrm{~mm})$ using a cu-

Table 1: Materials used in this study, manufacturer, and composition.

\begin{tabular}{|c|c|c|}
\hline CAD/CAM composites & \multicolumn{2}{|c|}{ Composition * } \\
\hline & Inorganic portion & Polymeric portion \\
\hline $\begin{array}{l}\text { Lava Ultimate } \\
\text { (3M ESPE, St. Paul, MN, USA) }\end{array}$ & $\begin{array}{l}80 \mathrm{wt} \%: 69 \% \text { silica } \mathrm{SiO}_{2}(20 \mathrm{~nm}), 31 \% \\
\text { zirconia } \mathrm{ZrO}_{2}(4-11 \mathrm{~nm}) \text { and zirconia+si- } \\
\text { lica clusters } \mathrm{ZrO}_{2} / \mathrm{SiO}_{2}(0.6-10 \mu \mathrm{m}) .\end{array}$ & $\begin{array}{c}20 \text { wt } \% \text { : bisphenol A-glycidyl methacrylate, } \\
\text { urethane dimethacrylate, bisphenol } \\
\text { A-diglycidyl methacrylate ethoxylated, } \\
\text { triethylene glycol dimethacrylate. }\end{array}$ \\
\hline $\begin{array}{l}\text { Brilliant Crios } \\
\text { (Coltène, Altstätten, Switzerland) }\end{array}$ & $\begin{array}{c}\text { 71wt \%: amourphous silica } \mathrm{SiO}_{2}(<20 \\
\mathrm{nm}) \text { and barium glass }(<1 \mu \mathrm{m}) .\end{array}$ & $\begin{array}{c}29 \mathrm{wt} \% \text { : bisphenol A-glycidyl methacrylate, } \\
\text { bisphenol A-diglycidyl methacrylate } \\
\text { ethoxylated, triethylene glycol } \\
\text { dimethacrylate. }\end{array}$ \\
\hline Materials for bonding protocols & \multicolumn{2}{|c|}{ Composition * } \\
\hline $\begin{array}{l}\text { Transbond }{ }^{\mathrm{TM}} \text { XT } \\
\text { 3M Unitek, Monrovia, CA, USA) }\end{array}$ & \multicolumn{2}{|c|}{$\begin{array}{l}\text { Etchant: } 40 \% \text { phosphoric acid gel. } \\
\text { Primer: trietylenoglicol-dimethetilacrylate }(45-55 \%) \text {, bisphenol A-glycidyl methacrylate } \\
(45-55 \%) . \\
\text { Adhesive: silane-treated quartz }(70-80 \%) \text {, bisphenol A-glycidyl methacrylate } \\
(10-20 \%) \text {, dichlorodimethylsinane reaction product with silica }(2 \%) .\end{array}$} \\
\hline $\begin{array}{l}\text { Condac Porcelana 5\% (FGM } \\
\text { Produtos Odont, Joinville, SC, } \\
\text { Brazil) }\end{array}$ & \multicolumn{2}{|c|}{ 5\% Hydrofluoric acid. } \\
\hline $\begin{array}{l}\text { Monobond Plus (IvoclarVivadent, } \\
\text { Liechteinstein) }\end{array}$ & \multicolumn{2}{|c|}{ Ethanol, 3-(trimethoxysilyl)propyl methacrylate, methacrylated phosphoric acid ester. } \\
\hline $\begin{array}{l}\text { Monobond Etch \& Prime (Ivoclar } \\
\text { Vivadent, Liechteinstein) }\end{array}$ & \multicolumn{2}{|c|}{$\begin{array}{l}\text { Ammoniun polyfluoride }+ \text { trimehtoxypropyl methacrylate }(15-25 \%) \text {, ethanol }+ \text { water } \\
\qquad(75-85 \%) .\end{array}$} \\
\hline
\end{tabular}

* Obtained from manufacturers' data and complemented according to Emsersann et al. 2019. 
tting machine (Isomet1000, Buehler, IL, USA). Sixty specimens were obtained per each CAD/CAM composite, which were embedded in self-curing acrylic resin (Jet, Lang Dental Manufacturing Co.; IL, USA). The specimen's upper surface was ground finished with 600 , 1000, 1200 and 2500-grit silicon paper (3M ESPE, MN, USA) under irrigation using a water-cooling machine (Leco Corporation, MI, USA) for $30 \mathrm{~s}$ and ultrasonically cleaned (15 min). The 60 specimens per each CAD/ CAM composite were randomized into 6 subgroups $(\mathrm{n}=10)$, according the treatments surface (Fig. 1), which

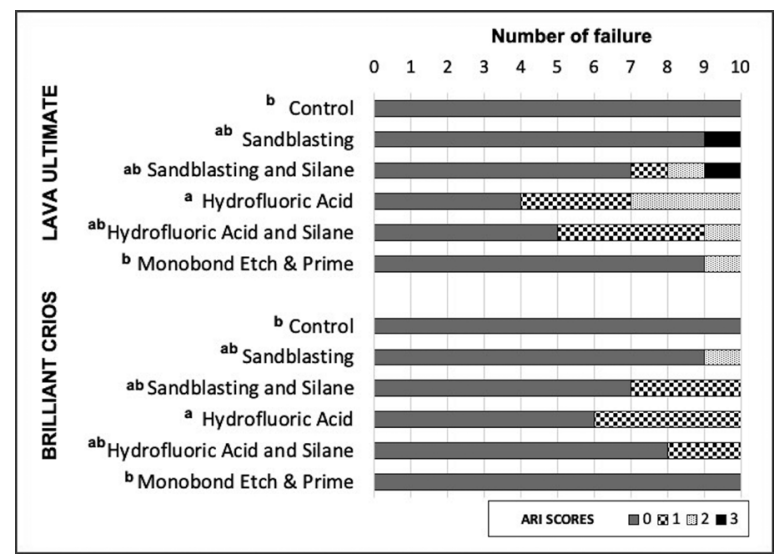

Fig. 1: Distribution of Adhesive Remnant Index (ARI) scores among the groups, and analysis according to Kruskal-Wallis $(p<0.01)$.

are described below. All the materials involving the surfaces treatment and bonding procedures, as well their manufacturers and composition are detailed in Table 1.

1. Control group (CTL): Etching with $40 \%$ phosphoric acid for $1 \mathrm{~min}$, rinsed for $1 \mathrm{~min}$ and air-dried. Then, Transbond XT primer was applied according to the manufacturer's instructions, and air-dried for $60 \mathrm{~s}$.

2. Sandblasting (SB): Sandblasting procedure (with aluminum oxide grain size $50 \mu \mathrm{m}$ [Kavo, Biberach, Germany] at two bars [30 psi] at $15 \mathrm{~mm}$ distance, until entire bonding surface appears matte [approximately 15 s]). After, etching with $40 \%$ phosphoric acid for $1 \mathrm{~min}$, rinsed for $1 \mathrm{~min}$ and air-dried. Transbond XT primer was applied according to the manufacturer's instructions, and air-dried for $60 \mathrm{~s}$.

3. Sandblasting and Silane (SBSL): Sandblasting procedure. After, etching with $40 \%$ phosphoric acid for $1 \mathrm{~min}$, rinsed for $1 \mathrm{~min}$ and air-dried. Then, a silane Monobond Plus was applied with a microbrush, allowing the reaction for $60 \mathrm{~s}$ and dried by gentle air blowing.

4. Hydrofluoric Acid (HF): Etching with 5\% hydrofluoric acid for $1 \mathrm{~min}$, rinsed for $1 \mathrm{~min}$ and air-dried. Then, Transbond XT primer was applied according to the manufacturer's instructions, and air-dried for $60 \mathrm{~s}$.
5. Hydrofluoric Acid and Silane (HFSL): Etching with $5 \%$ hydrofluoric acid for $1 \mathrm{~min}$, rinsed for $1 \mathrm{~min}$ and airdried. Then, a silane Monobond Plus was applied with a microbrush, allowing the reaction for $60 \mathrm{~s}$ and dried by gentle air blowing.

6. Monobond Etch \& Prime (MEP): Monobond Etch \& Prime was applied using a microbrush and agitated into the surface for $20 \mathrm{~s}$ using slight pressure, allowing to react for another $40 \mathrm{~s}$. Thoroughly rinsed until the green colour to be completly removed, and air-dried for $10 \mathrm{~s}$. After the surface treatment and bonding steps, the light-cure adhesive Transbond XT was used to bond the metal brackets to the specimens' surface. The adhesive was applied to the base of the stainless mandibular incisor metal brackets (3M Unitek, MN, USA). The brackets were seated on the CAD/CAM composites surface and a standardized constant of $100 \mathrm{~g}$ pressure using a customized metallic tool. All the steps were performed by the same operator. The bonding adhesive excess was carefully removed using an explorer and the light-curing $(20 \mathrm{~s}$ from the mesial and distal of the bracket) using a LED light-curing unit (Valo, $1000 \mathrm{~mW} / \mathrm{cm}^{2}$; Ultradent, UT, USA). The bonded specimens were stored in distilled water for $24 \mathrm{~h}$ at $37^{\circ} \mathrm{C}$.

- Shear bond strength and adhesive remnant index (ARI) The SBS was performed using a universal testing machine at $0.5 \mathrm{~mm} / \mathrm{min}$ (Instron Corp., MA, USA). The shearing wedge was positioned vertically at the bracket base (10). The values obtained were calculated in MPa (11). After debonding, the specimens were examined at the fractured area under 20X magnification (Olympus Optical, Tokyo, Japan) and the ARI was classified according to the Artun and Bergland: (12).

0 : no adhesive left on the CAD/CAM composite; 1: less than half of the adhesive left on the CAD/CAM composite;

2: more than half the adhesive left on the CAD/CAM composite;

3: all adhesive left on the CAD/CAM composite with distinct impression of the bracket mesh.

- Statistical Analysis

The data were submitted to a two-way analysis of variance (ANOVA) and Tukey's post hoc test considering two factors (CAD/CAM composite and surface treatment). The Kruskal-Wallis test was used to analyze the ARI scores. The statistical significance was set at $5 \%$. The software SPSS statistics 23.0 (IBM International Business Machine Corp., NY, USA) was used for all analyses.

\section{Results}

The two-way ANOVA revealed that the SBS was significantly affected by the CAD/CAM composite $(p<0.05)$ and by the surface treatment $(p<0.001)$, but there was no significant interaction between them $(p=0.125)$. The 
mean SBS values and standard deviation are shown in Table 2. Higher SBS was detected for BC in comparison with LU, regardless of surface treatment $(p<0.05)$. All the surface treatments promoted an increase of SBS values when compared with the CTL $(p<0.0001)$, for both the CAD/CAM composites. Highest SBS was detected by HF, followed by SB and SBSL $(p<0.0001)$. Intermediate SBS was detected by MEP and SB, and the lowest performance in the CTL $(p<0.0001)$.

The ARI scores are shown in Figure 1. The KruskalWallis analysis revealed that the ARI was affected only by the surface treatment $\left(\mathrm{X}^{2}(5)=20.710 ; p=0.001\right)$ and not by the CAD/CAM composite $\left(\mathrm{X}^{2}(1)=2.421\right.$; $p=0.12$ ), as shown in Table 3 . The ARI results also demonstrated that the adhesive failures between the $\mathrm{CAD} /$ CAM composite and adhesive were in all groups (Fig. 1). Comparing the surface treatments, significant differences were detected between HF and CTL $(p=0.03)$ and between HF and MEP $(p=0.014)$. Examination of the debonded surfaces showed no damage to the surfaces in any experimental group.

\section{Discussion}

The present study investigated the impact of the different $\mathrm{CAD} / \mathrm{CAM}$ composite and the surface treatment on the SBS of orthodontic metal brackets. It means a relevant clinical question, considering the fast introduction of novel materials in the market and there is a lack of specific bonding protocols, which vary widely according the different CAD/CAM materials $(2,6,8)$. According to the results, the CAD/CAM composite $(p<0.05)$ and the surface treatment $(p<0.0001)$ affected the SBS, leading to reject the null hypotheses.

In general, all the surface treatments significantly increased the SBS. Other studies demonstrated a contribution of the surface treatment in bonding protocols on $\mathrm{CAD} /$ CAM composites cementation (6-8). Also, the HF treatment increased the SBS of orthodontic brackets on LU (10), and in the polymer-infiltrated ceramic by Elsaka (11). The increase of SBS yielded by the HF, as well the other surface treatments in this study, could be explained by the higher surface roughness (13), which increases the impregnation of bonding agents (14).

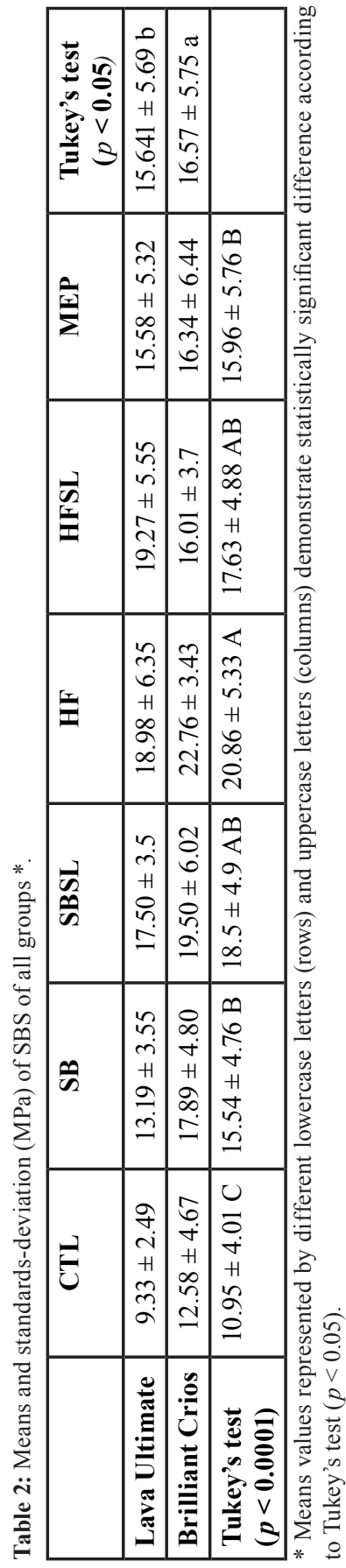

Table 3: Results of Kruskal-Wallis analysis with dependent variable according the distribution of ARI.*.

\begin{tabular}{|c|c|c|c|}
\hline \multicolumn{4}{|c|}{ Adhesive Remnant Index (ARI) } \\
\hline & Chi-Square $\left(\mathbf{X}^{2}\right)$ & degree of freedom & P value \\
\hline CAD/CAM composite & 2.421 & 1 & 0.12 \\
\hline Surface Treatment & 20.710 & 5 & 0.001 \\
\hline
\end{tabular}

*Statistically significant difference at $p<0.05$. 
Regarding all the treatments herein used, the highest SBS was detected by the HF, HFSL and SBSL. The etching with HF is highly recommended on glassy ceramics cementation, because it increases the surface roughness and the micromechanical retention by the bonding agent (15). The HF action mechanism creates an irregular etching pattern and a deeper glassy phase dissolution, not just superficially as in sandblasting. The etching HF is not based by the acid corrosion of ceramic's glassy matrix, but by the chemical interaction between the silicon particles on the glassy matrix and the HF fluoride ions (16). In case of CAD/CAM composites, the HF promotes etching of glassy particles, creating microporosities on the resin matrix, increasing surface energy and the wettability of bonding agents (17). Certainly, these surface modifications promoted by the HF allowed the deep primer infiltration, potentializing the mechanical interlocking and increase of SBS. The manufacturers of the CAD/CAM composites herein evaluated don't recommend the HF etching. However, it is worth mentioning that the concentration of the HF and time herein tested (5\% during $1 \mathrm{~min}$ ) had been considered a mild etching protocol, allowing an etching efficiency without compromising the mechanical properties and structural integrity of CAD/CAM composites (5). Also, the benefits promoted by the HF help us to understand the ARI results, in which the HF was significantly higher in comparison with the CTL.

The combination of the HF plus silane (HFSL) is considered the gold-standard surface treatment when dealing glassy ceramics (7), which lead to investigating this combination in our study. Silane is a bifunctional molecule with the siloxane group at one side, which needs to be hydrolyzed, and the methacrylate group at the other side, which forms covalent bonds with the glassy phase on ceramic materials and polymerize with resin-based materials (18). Even though the SB had been able to increase the SBS in comparison with the CTL, the results were lower than when combined with silane (SBSL). As the CAD/CAM composites contain a low amount of polymeric matrix and a high degree of conversion, a limited amount of free radicals are available for copolymerization with the new monomers from the primer (5). Thus, the bifunctional role of silane was important to interact chemically with the polymeric matrix from the CAD/CAM composite, increasing the SBS. Curiously, the silane didn't increase the SBS when combined with HF (HFSL). Maybe the deeper and complex etching pattern created by HF (16), was enough for an effective infiltration by the primer and micromechanical interlocking, increasing the SBS.

Following the simplification concept on ceramics bonding protocol, the self-etching ceramic primer Monobond Etch \& Prime (MEP) was developed to combine the etching acid and silanization in one step. The main active ingredients are the ammonium polyfluoride, which wor$\mathrm{ks}$ in etching, and the trimethoxypropyl methacrylate in the silanization $(5,9)$. In this study, MEP showed results that were statistically similar to other groups that combined the increase of roughness plus silanization (SBSL and HFSL), which means that MEP is a clinically interesting product. Promising results were shown on ceramic materials, promoting a less-aggressive etching pattern in comparison with HF, and similar bond strength in comparison with the gold-standard HFSL $(5,19)$. Thus, a more superficial etching depth in MEP could result in less adhesive micromechanical interlocking and could cause the significantly lower ARI results when compared with HF. The benefits of MEP were also evaluated on the SBS of orthodontic brackets on a zirconia surface (20). However, the impact of surface treatment using MEP on CAD/ CAM composites still have not been evaluated.

Even though the resins herein tested are classified as CAD/CAM composites with dispersed fillers (2), they have different composition in terms of the amount and type of inorganic filler. Thus, a significant difference between them could be expected. Contrary to our results, Buyuki and Kucukekenci (10) found a significant effect on SBS for the factor CAD/CAM material and a non-significant by the etching acid. However, in this study only LU was evaluated, and it was compared with a glassy ceramic and a hybrid ceramic, which vary considerably in type and material properties, as well as in response to different surface treatment protocols (7).

A higher bond strength was also detected for $\mathrm{BC}$ when compared with LU (6). Nonetheless, in this study, the factor resin was not explored. BC shows $29 \mathrm{wt} \%$ of organic matrix and $71 \mathrm{wt} \%$ of inorganic fillers containing amorphous silica and barium glass. However, LU shows $20 \mathrm{wt} \%$ organic matrix and $80 \mathrm{wt} \%$ of inorganic fillers containing silica, zirconia and silica-zirconia nanoclusters. A higher percentage of an organic matrix could contribute for the chemical bonding mechanism, and copolymerization between the uncured organic matrix from the primer/adhesive and the cross-linked CAD/CAM composite (21). As previously mentioned, the microstructure and high degree of conversion reduce the copolymerization ability between both of the organic matrices (2). This indicate that bonding to the CAD/CAM composites highly depends on micromechanical interlocking, and in fact was confirmed by the high statistically significance detected for the "surface treatment" factor. Another interesting point to considered, is the type of inorganic filler present in BC, such as the amorphous silica and barium glass, which allowed the etching acid by the HF or MEP. Although a significant percentage of inorganic composition in LU has zirconia particles, which increases the resilience and wear resistance, they are not etched by acids. This results in less micromechanical interlocking by the primer and adhesive. 
The ARI results predominantly demonstrated score 0 (between the CAD/CAM composite and the adhesive) and it is according to the literature (11), although adhesive failures at the CAD/CAM composite-adhesive interface would be most favorable to avoid the CAD/ CAM composite fractures during debonding $(11,22)$. There was no damage to the debonded surface in any group. Also, it is worth mentioning that the SBS results in the present study were higher than what is considered sufficient for clinical applications (5.8 - 7.8 $\mathrm{MPa})(23)$. This means that the CAD/CAM composites and the surface treatments herein tested can be considered reliable for clinical applications.

\section{Conclusions}

Based on the current results, it can be concluded that the SBS of orthodontic brackets is highly influenced by the surface treatment, followed by the CAD/CAM composites. All the surface treatment herein tested positively contributed to the SBS. Thus, when the adhesion of metal brackets is performed on CAD/CAM composites, a surface treatment should be preconized by the significant contribution on SBS and could result in less adhesive failures during orthodontic treatment.

\section{References}

1. Kollmuss M, Kist S, Goeke JE, Hickel R, Huth KC. Comparison of chairside and laboratory CAD/CAM to conventional produced all-ceramic crowns regarding morphology, occlusion, and aesthetics. Clin Oral Investig. 2016;20:791-97.

2. Mainjot AK, Dupont NM, Oudkerk JC, Dewael TY, Sadoun MJ. From Artisanal to CAD-CAM Blocks: State of the Art of Indirect Composites. J Dent Res. 2016;95:487-95.

3. Abdelnaby YL. Effects of cyclic loading on the bond strength of metal orthodontic brackets bonded to a porcelain surface using different conditioning protocols. Angle Orthod. 2011;81:1064-69.

4. Abu Alhaija ES, Abu AlReesh IA, AlWahadni AM. Factors affecting the shear bond strength of metal and ceramic brackets bonded to different ceramic surfaces. Eur J Orthod. 2010;32:274-80.

5. Colombo LDA, Murillo-Gomez F, De Goes MF. Bond Strength of CAD/CAM Restorative Materials Treated with Different Surface Etching Protocols. J Adhes Dent. 2019;21:307-17.

6. Emsermann I, Eggmann F, Krastl G, Weiger R, Amato J. Influence of Pretreatment Methods on the Adhesion of Composite and Polymer Infiltrated Ceramic CAD-CAM Blocks. J Adhes Dent. 2019;21:43343.

7. Peumans M, Valjakova EB, De Munck J, Mishevska CB, Van Meerbeek B. Bonding Effectiveness of Luting Composites to Different CAD/CAM Materials. J Adhes Dent. 2016;18:289-302.

8. Spitznagel FA, Vuck A, Gierthmühlen PC, Blatz MB, Horvath SD. Adhesive Bonding to Hybrid Materials: An Overview of Materials and Recommendations. Compend Contin Educ Dent. 2016;37:630-7.

9. Monobond Etch and Prime. 2015. http://www.ivoclarvivadent.com. Accessed May 11, 2020.

10. Buyuk SK, Kucukekenci AS. Effects of different etching methods and bonding procedures on shear bond strength of orthodontic metal brackets applied to different CAD/CAM ceramic materials. Angle Orthod. 2018;88:221-6.

11. Elsaka SE. Influence of surface treatments on bond strength of metal and ceramic brackets to a novel CAD/CAM hybrid ceramic material. Odontology. 2016;104:68-76.
12. Artun J, Bergland S. Clinical trials with crystal growth conditioning as an alternative to acid-etch enamel pretreatment. Am J Orthod. 1984;85:333-40.

13. Papadopoulos K, Pahinis K, Saltidou K, Dionysopoulos D, Tsitrou E. Evaluation of the Surface Characteristics of Dental CAD/ CAM Materials after Different Surface Treatments. Materials (Basel). 2020;13:981.

14. Gilbert S, Keul C, Roos M, Edelhoff D, Stawarczyk B. Bonding between $\mathrm{CAD} / \mathrm{CAM}$ resin and resin composite cements dependent on bonding agents: three different in vitro test methods. Clin Oral Investig. 2016;20:227-36.

15. Brentel AS, Ozcan M, Valandro LF, Alarca LG, Amaral R, Bottino MA. Microtensile bond strength of a resin cement to feldpathic ceramic after different etching and silanization regimens in dry and aged conditions. Dent Mater. 2007;23:1323-31.

16. Tian T, Tsoi JK, Matinlinna JP, Burrow MF. Aspects of bonding between resin luting cements and glass ceramic materials. Dent Mater. 2014;30:e147-62.

17. Ramakrishnaiah R, Alkheraif AA, Divakar DD, Matinlinna JP, Vallittu PK. The Effect of Hydrofluoric Acid Etching Duration on the Surface Micromorphology, Roughness, and Wettability of Dental Ceramics. Int J Mol Sci. 2016;17:822.

18. Matinlinna JP, Lung CYK, Tsoi JKH. Silane adhesion mechanism in dental applications and surface treatments: A review. Dent Mater. 2018;34:13-28.

19. Murillo-Gomez F, Palma-Dibb RG, De Goes MF. Effect of acid etching on tridimensional microstructure of etchable CAD/CAM materials. Dent Mater. 2018;34:944-55.

20. Franz A, Raabe M, Lilaj B, Dauti R, Moritz A, Müßig D, et al. Effect of two different primers on the shear bond strength of metallic brackets to zirconia ceramic. BMC Oral Health. 2019;19:51.

21. Tezvergil A, Lassila LV, Vallittu PK. Composite-composite repair bond strength: effect of different adhesion primers. J Dent. 2003;31:521-5.

22. Saraç Y, Külünk T, Elekdağ-Türk S, Saraç D, Türk T. Effects of surface-conditioning methods on shear bond strength of brackets bonded to different all-ceramic materials. Eur J Orthod. 2011;33:667-72. 23. Reynolds IR. A Review of Direct Orthodontic Bonding. Br J Orthod. $1975 ; 2: 171-8$.

\section{Conflict of interest}

The authors declare that there is no conflict of interest. 